\title{
UN ANÁLISIS DE RACIONALIDAD LEGISLATIVA COMPARADA: ESPAÑA (2005) Y PERÚ (2014) SOBRE EL DEBATE EN TORNO A LA UNIÓN CIVIL Y MATRIMONIO ENTRE PERSONAS DEL MISMO SEXO
}

\author{
AN ANALYSIS OF COMPARATIVE LEGISLATIVE RACIONALITY: \\ SPAIN (2005) AND PERU (2014) ABOUT THE DEBATE AROUND \\ THE CIVIL UNION AND SAME-SEX MARRIAGE
}

\author{
José Enrique Sotomayor Trelles ${ }^{\star}$ \\ Pontificia Universidad Católica del Perú
}

\begin{abstract}
This article studies the parliamentary debate over the approval of a legislative change on the Spanish Civil Code, which gave permission to Same-Sex marriages since 2005. The author tries to approach to Congress debates as complex processes, in which different levels of rationality (as of irrationality) are intersperse in the argumentations of senators and deputies.
\end{abstract}

In order to clarify this confounding scenario, the tools of 'legisprudential studies' or legislative rationality are of utmost importance, because they allow us to group the reasons in typologies, and to analyze possible counter-arguments or voids relating to supporting evidence. That is why the approach of this work, in relation to the substance of the matter, is neutral and rather points to demonstrate the immense methodological potential of legislative rationality models, and how these could contribute to illuminate the ongoing discussion on our country.

KEYWORDS: Legislative rationality; legisprudence studies; levels of parlament rationality; same-sex marriage; civil union.
El presente artículo realiza un estudio sobre el debate parlamentario en torno a la aprobación de una modificación al Código Civil español, que permitió el matrimonio entre personas del mismo sexo desde el 2005. En ese sentido, el autor busca aproximarse a los debates parlamentarios como procesos complejos, en donde los distintos niveles de racionalidad (e irracionalidad) están entremezclados en las argumentaciones de senadores y diputados.

Para aclarar el confuso panorama, las herramientas de los "estudios legisprudenciales" o de racionalidad legislativa resultan fundamentales, pues permiten agrupar a los argumentos en tipologías, y pasar a analizar posibles contraargumentos o vacíos en cuanto a evidencia de respaldo. Es por ello que la perspectiva del presente trabajo es neutral en cuanto al fondo del asunto, y apunta más bien a mostrar el gran potencial metodológico de modelos de racionalidad legislativa, y cómo estos contribuirían a aclarar el debate en curso en nuestro país.

Palabras CLAVE: Racionalidad legislativa; Estudios legisprudenciales; niveles de racionalidad parlamentaria; matrimonio entre personas del mismo sexo; unión civil.

\footnotetext{
Abogado. Magíster en Filosofía por la Pontificia Universidad Católica del Perú (PUCP). Magister en Global Rule of Law and Constitutional Democracy por la Università degli Studi di Genova. Magíster en Economía por la Universidad Mayor de San Marcos.
}

Nota del Editor: El presente artículo fue recibido por el Consejo Editorial el día 02 de marzo del 2017, y aceptado el día 05 de Agosto del 2017. 


\section{INTRODUCCIÓN}

El presente trabajo se aboca a un análisis de los debates, en el Congreso y luego en el Senado, respecto de la aprobación de una modificación en el artículo 44 del Código Civil español, mediante la cual se permitiría contraer matrimonio a personas del mismo sexo. Para el logro de un adecuado análisis, se ha dividido la presentación en dos partes: en la primera realizamos una ampliación del marco conceptual. Esta ampliación busca incorporar y adecuar algunas categorías teóricas provenientes de los estudios legisprudenciales o de racionalidad legislativa. La segunda parte aplica las nociones conceptuales previamente presentadas al estudio de caso.

Al igual que un texto reciente de Oliver-Lalana ${ }^{1}$, nos limitaremos al análisis de la transcripción de los debates parlamentarios. Sin embargo, es necesario apuntar que dicha restricción parte solo del carácter indiciario de este trabajo. Una interesante línea de investigación para los estudios de racionalidad legislativa consistiría en incorporar intervenciones públicas de los parlamentarios en medios de comunicación, mítines políticos y reuniones en ámbitos más académicos como organizaciones, universidades o think tanks. Este estudio transversal podría permitir identificar patrones de adaptación frente a distintos tipos de público por parte de los oradores. Esta vía de investigación resultaría muy fructífera para la retórica. Por otra parte, nos permitiría identificar el nivel de porosidad -apertura cognitiva, en términos luhmannianos- de los parlamentarios frente a argumentos provenientes de diversos sectores de la sociedad civil.

\section{BREVE MARCO TEÓRICO}

La ampliación teórica que proponemos se basa en dos elementos, uno sistémico y otro asociado a la dimensión de legitimidad del parlamento. Ambos, al juntarse, conforman una explicación satisfactoria sobre las razones por las cuales es deseable que los parlamentos se involucren en amplios debates en los que se discuten propuestas legislativas.

El primer elemento tiene que ver con la función de descarga que juega la legislación en el sistema social del derecho. Siguiendo la distinción luhmanniana entre sistema y entorno ${ }^{2}$, debemos comenzar a estudiar al derecho como un subsistema social, diferenciado por un código compartido a partir del cual opera. [El enfasis es nuestro].

El código binario del derecho reduce la enorme complejidad del mundo a una variable booleana, la de "lo jurígeno/no jurígeno" ${ }^{3}$. Pero además el sistema se comunica con el entorno a través de un complejo entramado de filtros por los cuales un conjunto de demandas, provenientes de otros sistemas, se filtran al derecho ${ }^{4}$. En principio, la interrelación entre derecho y sociedad se produce mediante la intermediación del sistema político. El parlamento, en el modelo ideal de división de poderes, recoge las demandas sociales y las filtra en el código político. Estas demandas se canalizan en diversos tipos de medidas, una de las cuales son las leyes. Lo anterior se puede vincular, además, con la categorización de niveles de racionalidad en Atienza ${ }^{5}$. El sistema parlamentario transforma razones y justificaciones, principalmente, en los niveles R3, R4 y R5, en razones y justificaciones que deben cumplir con condiciones de coherencia jurídico formal (R2) y claridad comunicativa (R1). Ahora bien, esta reconstrucción no resulta del todo fidedigna.

En el plano ideal, los parlamentos deberían transformar demandas sociales en pretensiones políti-

OLIVER-LALANA, Daniel. "Normas y razones: un estudio sobre argumentación legislativa”. En: GRÁNDEZ, Pedro y Félix MORALES. "La argumentación jurídica en el Estado Constitucional”. Lima: Palestra. 2013. pp. 491-525.

2 Véase LUHMANN, Niklas. "Operational closure and structural coupling: The differentiation of the legal system". En: Cardozo Law Review 13. 1992. pp. 1419-1441. Asimismo, cabe recordar que los componentes de los sistemas en la teoría de sistemas de Luhmann no son seres humanos sino comunicaciones. Véase GARCÍA AMADO, Antonio. "La filosofía del derecho en Habermas y Luhmann". Bogotá: Universidad Externado de Colombia. 1997. En especial, pp. 135-140.

3 Aquí se debe hacer una precisión. Luhmann hace alusión a dos niveles distintos de códigos binarios que son relevantes para el sistema del derecho, pero que no se deben confundir: en primer lugar, tenemos al código de lo justo y lo injusto, que es de donde el derecho obtiene las expectativas normativas que desea asegurar contrafácticamente. Sin embargo, sería una confusión concluir que el código de la justicia es aquel que es inmediatamente relevante para el derecho, pues este adapta a y se alimenta del código de la justicia para establecer su propio y nuevo código. Es a este segundo nivel que se encuentra lo jurígeno y lo no jurígeno (que hemos traducido de las expresiones inglesas lawful y unlawful, aunque en otros lugares se ha traducido al código del derecho como "legal/ilegal"). Confundir los planos de la justicia y el derecho, implica una confusión de dos sistemas sociales distintos, y ello es algo con lo que seguramente Luhmann no estaría de acuerdo. Véase: LUHMANN, Niklas. "Law as a social system". En: Northwestern University Law Review 83. 1988-1989. pp. 136-150; en especial p. 140. Adicionalmente, la función de las normas jurídicas en la teoría de Luhmann es la de estabilizar contrafácticamente a las expectativas de comportamiento. El instrumento para ello es la fuerza. Véase GARCÍA AMADO, Antonio. Óp. Cit. pp. 171-184

4 Por ejemplo, un tipo de demandas que se filtran al derecho son las demandas sociales. Véase FERNANDES, Celso. "Protestas sociales dentro del derecho". Lima: Fondo Editorial PUCP e Hipocampo. 2012.

5 ATIENZA, Manuel. "Contribución a una teoría de la legislación". Madrid: Cívitas. 1997. p. 27 y siguientes 
cas jurídicamente coherentes y compatibles con un ordenamiento legal y constitucional. Sin embargo, esta pretensión no siempre se cumple en la realidad y algunas veces los parlamentarios -en especial cuando saben que en sus ordenamientos existen cortes que ejercen control constitucional y que pueden enmendar sus errores- actúan de forma más irresponsable, aprobando normas de dudosa compatibilidad constitucional. Este fenómeno es al que se refiere Tushnet con el nombre de judicial overhang ${ }^{6}$.

Oliver-Lalana señala que, asimismo, en los cinco niveles de racionalidad legislativa de Atienza, se nota una omisión de la dimensión constitucional. Ello es así en la medida que restringir los argumentos constitucionales a cuestiones jurídico-formales y sistemáticas, reduce el espacio de discusión al de una dogmática sobre la interpretación de normas de la Constitución (veremos que esto cumple un rol importante en el debate sobre el artículo 32 de la Constitución española, que vamos a analizar), coherente con una teoría de corte más positivistaformalista, pero insuficiente para dar cuenta de la dimensión de legitimidad constitucional.

Vemos entonces que el sistema jurídico se comunica de forma indirecta con las demandas sociales, económicas y políticas. En términos luhmannianos, el sistema es operativamente cerrado pero cognitivamente abierto. El elemento de apertura cognitiva viene asociado a una característica compartida por todo sistema: la autopoiesis.

El término es importado por Luhmann del vocabulario de algunos biólogos y hace alusión a la capacidad de perpetuación de las condiciones de existencia por parte de un sistema. Así como los organismos biológicos son capaces de perpetuar su existencia mediante un conjunto de procesos físico-químicos de preservación de la vida, así también los sistemas (económico, político o jurídico) se hacen porosos al entorno para preservar su existencia en el tiempo.

Ahora bien, frente a un funcionamiento defectuoso o ralentizado en el sistema político, el vínculo del derecho con el sistema social se dificulta. En el largo plazo, esta ralentización no sólo perjudica a la legitimidad del sistema, sino a su existencia misma. Por ello, el sistema jurídico desarrolla mecanismos internos de adaptación, uno de los cuales es el sistema de reformas normativas ${ }^{7}$.

Aquí, los parlamentos cumplen un rol gravitante. Mediante la actualización de la legislación a los nuevos tiempos, el derecho se hace poroso a demandas sociales o a procesos de modernización, liberalización o simple cambio social. Por ello, cuando mayorías parlamentarias bloquean grandes reformas normativas durante largos periodos de tiempo, dejan al sistema sin capacidad de incorporar o adaptar demandas sociales al sistema jurídico, lo que genera una peligrosa tendencia al recalentamiento ${ }^{8}$. Tenemos entonces un primer elemento: los parlamentos son, en sí mismos, una institución dual pues permiten la reproducción del subsistema jurídico, a la vez que la apertura cognitiva a demandas no sólo sociales sino también provenientes de los demás subsistemas, como el económico.

El segundo elemento viene dado por la dimensión de legitimidad ${ }^{9}$ sistémica del derecho. Dicha dimensión ha sido defendida por Habermas, frente a teorizaciones del derecho como la weberiana, que enfatizan en la técnica del derecho y propugnan la aparición de una suerte de "fe en la legalidad", que se convierte en un simple principio de positividad: Satzungprinzip ${ }^{10}$. La dimensión de legitimidad del

6 TUSHNET, Mark. "Is congress capable of conscientious, responsible constitutional interpretation?". En: Boston University Law Review 89. 2009. p. 499. Contrastar con SIECKMANN, Jan. "Legislation as Implementation of Constitutional Law: A Foundation for the Demand of Legislative Rationality". En: WINTGENS, L.J. y Daniel OLIVER-LALANA. "The rationality and Justification of Legislation". Suiza: Springer. 2013.

7 Confrontar con la función que el propio Luhmann atribuye a la argumentación jurídica y a técnicas como la de la ponderación. LUHMANN, Niklas. "Legal argumentation: an analysis of its form”. En: The Modern Law Review 58. 1996. pp. $285-298$.

8 Para los problemas y críticas que enfrentó el originalismo (en el ámbito de la adjudicación) con este intento que podríamos llamar -con Sager- "parsimonioso", véase SAGER, Lawrence. "Juez y democracia". Madrid: Marcial Pons, 2007. En especial capítulos II y III. Es importante notar que, en el debate que analizaremos, la facción progresista critica a los conservadores por no haber adecuado el derecho a los nuevos tiempos, aun cuando tuvieron muchas oportunidades para hacerlo. El congresista Villarrubia Mediavilla lo señala con especial claridad: "[...] se retratan un poquito [...] y hablan de regular las parejas de hecho. ¿No les parece un poco tarde? Creo que se han rechazado hasta 31 iniciativas por mayoría absoluta. Como llegan tarde, como siempre, y obligados, ahora para intentar boicotear y paralizar este procedimiento [...], hablan de impulsar esas uniones de hecho sobre las que han votado en contra hasta hace cuatro días, impidiendo que la voluntad del resto de la Cámara pudiera abrirse camino". "Debates en el Congreso".17 de marzo de 2005. pp. 3787. El citado congresista critica a la oposición por no haber propuesto la unión civil antes, y recién usarla como argumento cuando se veían acorralados con la opción matrimonial.

9 OLIVER-LALANA, Daniel. "Rational Lawmaking and Legislative Reasoning in Parliamentary Debates". En: WINTGENS, L.J., Daniel OLIVER-LALANA. "The rationality and Justification of Legislation". Óp. cit. pp. 136-137.

10 HABERMAS, Jürgen. "Teoría de la acción comunicativa”, tomo I. Madrid: Taurus. 1999. pp. 342 y siguientes. 
derecho asocia a todo el sistema jurídico con la noción de consenso de fondo sobre la pertinencia de sus instituciones. En este punto, podemos establecer algunas semejanzas con los principios de legisprudencia propuestos por Wintgens ${ }^{11}$, en la medida que los mismos parten del requisito de que cualquier medida legislativa cuente con una justificación satisfactoria, pues en caso contrario representa una restricción ilegítima de libertad.

Nótese la operación habermasiana en este punto. La idea de consenso subyace como el ideal de toda comunicación orientada al entendimiento ${ }^{12}$, y en el marco del sistema del derecho ello implica que todo proceso de debate parlamentario requiere de una argumentación validable frente a cualquier hablante o participante que compartiera la orientación hacia el entendimiento. Ello nos lleva a una operación que supone el fin de cualquier pretensión irracionalista: frente a cualquier debate aparentemente aporético, debido a la polarización ex ante que genera entre bancadas parlamentarias, se hace necesario un ejercicio de argumentación, un proceso de dar y recibir razones, orientado hacia el entendimiento.

La autonomía de la decisión parlamentaria viene dada por la expresión de un consenso sobre cuestiones que ex ante a la decisión se mostraban polémicas. Aquí se busca la construcción de un argumento que sea reconocible por cualquier participante comunicativamente racional. Este será, pues, nuestro segundo elemento y lo podemos reformular así: los parlamentos realizan argumentaciones prácticas y morales en aquellos casos en los que el derecho se muestra insuficiente frente al cambio social, o requiere un cambio pues ha devenido en inoperante o injusto, y se requiere una argumentación orientada al consenso.

\section{ANÁLISIS DE CASO: DEBATES SOBRE LA PROPUESTA DE LEY DE MATRIMONIO EN- TRE PERSONAS DEL MISMO SEXO}

El caso de análisis consiste en la propuesta del 2005 de modificación del artículo 44 del Código Civil español, que desencadenó finalmente en su aprobación desde el 3 de julio del 2005. Dicha modificación suponía la adición de un segundo párrafo que señala, según el ministro de justicia, lo siguiente: "El matrimonio tendrá los mismos requisitos y efectos cuando ambos contrayentes sean del mismo o distinto sexo ${ }^{13 \prime}$. Frente a esta propuesta de modificación se presentaron dos enmiendas a la totalidad, tanto en sede del Congreso, como del Senado.

11 WINTGENS, Luc J. "Legisprudence. Practical Reason in Legislation". Aldershot: Ashgate. 2012. https://doi.org/10.4324/9781315592145

12 Las nociones de consenso y de decisión basada en autonomía son importantes para comprender la extensión y pretensión de nuestra propuesta. Las mismas tienen dos dimensiones que creemos necesario resaltar: una epistémica y una performativa. La dimensión epistémica exige que cada parlamentario se comporte como un hablante racional que sólo puede ser convencido por las mejores razones. Sin embargo, su búsqueda no es un monólogo de construcción de argumentos, sino un diálogo y discusión en el que se incorporan partes que pueden y quieren aportar argumentos. Nino refleja bien ello cuando plantea la postura E2 en su categorización de tesis epistemológicas respecto de la verdad moral. Aunque su caracterización de Habermas resulta exagerada, la posición que él defiende recoge varios de los elementos que ahora nos interesa resaltar. Así, para Nino: 'La discusión y la decisión intersubjetiva constituyen el procedimiento más confiable para tener acceso a la verdad moral, pues el intercambio de ideas y la necesidad de ofrecer justificaciones frente a otros no sólo incrementa el conocimiento que uno posee y detecta defectos en el razonamiento, sino que ayuda a satisfacer el requerimiento de atención imparcial a los intereses de los afectados. Sin embargo, esto no excluye la posibilidad de que a través de la reflexión individual alguien pueda tener acceso al conocimiento de soluciones correctas, aunque debe admitirse que este método es mucho menos confiable que el colectivo, debido a la dificultad de permanecer fiel a la representación de los intereses de otros y ser imparcial".

Por su parte, el elemento performativo requiere un especial tipo de actitud o predisposición en el hablante. Josep Aguiló ha desarrollado, recientemente, una categorización de modos de debatir que nos puede ser de utilidad en este punto. Él agrupa a las actitudes en cuatro tipos ideales, que se ubican en un continuo que va desde el conflicto, en su forma más extrema, hasta la cooperación "ingenua". En esta categorización, el consenso ocupa el lugar más elevado pues exige un tipo de debate cooperativo y una dimensión performativa o actoral. La dimensión performativa consiste en una actitud del hablante que le obliga a buscar una aceptación de las razones por parte de todos sus interlocutores. Aquí Aguiló yerra atribuyendo una suerte de derecho retórico al veto (como una manifestación de la dimensión de poder presente en la deliberación). Su yerro consiste en extrapolar una regla procedimental (la de veto) a un tipo de debate que tiene una carga epistémica mucho más fuerte que su dimensión procedimental. Para incorporar estas reflexiones a nuestro debate diríamos que los hablantes no buscan un procedimiento sobre el momento de hablar, los tiempos y las formas, sino que se comprometen con una empresa cooperativa y unánimemente aceptada de acuerdo. En este modelo, el juez cumple el rol de acercamiento de las partes y de creación de una disposición al diálogo racional.

Ahora bien, el modelo -con sus exigencias epistémicas y performativas- funciona como un ideal regulativo, como un modelo normativo al cual se deben acercar las situaciones reales. Evidentemente los casos reales se acercan más a debates con argumentos falaces pero convincentes, y a situaciones en las cuales quienes discuten pretenden sólo convencer al auditorio, y no están comprometidos con una búsqueda colectiva de un mejor argumento. Sin embargo, ello no obsta para que la propuesta siga siendo útil y potente como estándar contrafáctico de crítica. Véase NINO, Carlos. "La Constitución de la democracia deliberativa". Barcelona: Gedisa, 2009. pp. 161. Asimismo, AGUILÓ, Josep. "Cuatro modos de debatir". En: DOXA - Cuadernos de Filosofía del Derecho 36. 2013. pp. 211-227.

13 "Debate en el Congreso".17 de marzo 2005. p. 3769. 
Cuadro 1

Resumen de argumentos usados en los debates en el Congreso y en el Senado, en relación de la ley que modifica el Código Civil, para incorporar la figura del matrimonio entre personas del mismo sexo

\begin{tabular}{|c|c|c|}
\hline R1 & $\begin{array}{l}\text { - La Constitución española no lo prohíbe en su artículo 32a } \\
\text { (el mismo quería enfatizar en la igualdad entre hombres } \\
\text { y mujeres, pero no prohibir otras formas de matrimo- } \\
\text { nio). En este argumento, se propugna una interpretación } \\
\text { dinámica -diacrónica en términos saussureanos- de la } \\
\text { palabra "matrimonio". La misma pasa a convertirse en } \\
\text { una institución cambiante en el tiempo. }\end{array}$ & $\begin{array}{l}\text { - El matrimonio es inconstitucional de acuerdo al artícu- } \\
\text { lo } 32 \text { de la Constitución española, que solo permite el } \\
\text { mismo entre hombre y mujer. Por oposición a la inter- } \\
\text { pretación dinámica de la palabra "matrimonio", aquí se } \\
\text { propugna una interpretación estática (puramente sin- } \\
\text { crónica) sobre el significado }{ }^{14} \text {. }\end{array}$ \\
\hline & \multicolumn{2}{|c|}{$\begin{array}{l}\text { - Argumento de consenso: Cualquiera de los proyectos -ya sea matrimonio o el de unión civil- llena una laguna del } \\
\text { ordenamiento español }{ }^{15} \text {. }\end{array}$} \\
\hline
\end{tabular}

14 Véase, en especial, la crítica de Díaz Tejera en el Senado: “[...] Si se dice que ahora se busca [...] la equiparación en materia de derecho sucesorio, de derecho tributario, en seguros, en el momento de contratar una hipoteca o en el momento de acceder a servicios públicos, si se dice todo esto, ¿por qué cuando se dice: podemos equipararlos en todo, ¿Existe ese temor al uso de las palabras? ¿Es sólo un debate nominal? ¿Es un debate en torno al "nomen", a llamarlo matrimonio? No enciendo qué sentido tiene". "Debates en el Senado-Pleno". p. 2508. Ahora bien, este argumento que podríamos llamar "de aguijón semántico" aparece algunas veces entremezclado con una supuesta función natural de la institución del matrimonio. Veamos la siguiente cita de senador Casas i Bedós, quien trae a colación una cita de Lionel Jospin: "El matrimonio es en su origen y en tanto que institución la unión de un hombre y una mujer. Esta definición no obedece al azar. No remite, en primer lugar, a una inclinación sexual, sino a la dualidad de sexos que caracteriza nuestra existencia y que constituye la condición de procreación y en consecuencia, de la continuación de la Humanidad". "Debates en el Senado-Pleno". p. 2500. Cuando el argumento aparece en esta forma, la prohibición de matrimonio de personas del mismo sexo adopta una forma teleológica (R4 en la propuesta de Atienza). La restricción aparece para preservar una institución importante para la sociedad.

15 En la siguiente cita, el argumento sistemático se complementa con un argumento democrático y quien lo formula es un congresista opositor a la propuesta de matrimonio, Tome Pardo: "Señorías, deberíamos abordar la regulación jurídica de la convivencia de parejas homosexuales con un gran acuerdo, porque de esa manera estaríamos dando una solución a un vacío legal existente y, además, recogeríamos el sentir mayoritario de la sociedad española". Debate en el Congreso. p. 3776.

16 Véase en especial la intervención de la senadora Vindel López en los debates del Senado. Ella recuerda el caso de Ontario, Canadá, en donde la institución del matrimonio entre personas del mismo sexo supuso un gran proceso de reformas normativas que incrementaron la inseguridad en el tráfico jurídico. "Debates en el Senado-Pleno". pp. 2521 y 2522.

17 Duran i Lleida: "[...] Y mucho menos voy a ampararme en sus argumentos a la Constitución europea. Yo fui de los que se mojaron públicamente para la defensa de la Constitución europea, la voté y no comparto en absoluto la interpretación que usted hace de la misma. Entre otras cosas, usted mismo señala que lo remite a las legislaciones de los Estados y eso es algo que forma parte de otro documento o principio de la Constitución europea que es el principio de subsidiariedad. Por tanto, no es cierto que la Constitución europea abra o no el terreno, lo que hace es permitir que cada Estado haga lo que crea oportuno, cosa que era exactamente igual antes que después de la Constitución europea". "Debate en el Congreso". p. 3773.

18 Tome Pardo: "La iniciativa no es pacífica, sino que provoca confrontación y crispación social; no cuenta con el respaldo mayoritario de la sociedad y ese concepto de ciudadanía, al que ustedes les gusta tanto aludir, se traduce en gobernar para muy pocos". "Debate en el Congreso". p. 3777. 


\begin{tabular}{|c|c|c|}
\hline R4 & $\begin{array}{l}\text { - El proyecto busca como finalidad la equiparación de de- } \\
\text { rechos, pero también simbólica (legislación simbólica), } \\
\text { entre personas heterosexuales y homosexuales. Para el } \\
\text { logro de esta finalidad, solo queda ir por la medida que } \\
\text { ofrece una real equiparidad. } \\
\text { - El proyecto busca que las parejas homosexuales puedan } \\
\text { adoptar niños. Ello debido a que no existe evidencia } \\
\text { científica contundente sobre el daño que podría ser cau- } \\
\text { sado a los niños. }\end{array}$ & $\begin{array}{l}\text { - La finalidad de equiparación es defendible también por } \\
\text { los críticos al proyecto. Sin embargo, se sostiene que } \\
\text { existen vías alternativas más idóneas para lograr la fina- } \\
\text { lidad de la igualdad. }{ }^{19} \\
\text { - No se debe permitir el matrimonio porque con ello se } \\
\text { abriría la puerta a la adopción, y este no es un derecho } \\
\text { de los adoptantes sino de los niños adoptados. } \\
\text { - El proyecto no va a lograr cambiar a las prácticas discri- } \\
\text { minatorias, porque las leyes no pueden cambiar patro- } \\
\text { nes de conducta. }{ }^{20}\end{array}$ \\
\hline R5 & $\begin{array}{l}\text { - Defensa del valor de la igualdad }{ }^{21} \\
\text { - Defensa del valor de la libertad: variantes del argumen- } \\
\text { to: a) la libertad de amar, b) la libertad para buscar la } \\
\text { felicidad }^{22} \text {. }\end{array}$ & $\begin{array}{l}\text { - Defensa del valor de la cohesión de la comunidad, el va- } \\
\text { lor de la familia y el matrimonio. } \\
\text { - Defensa del valor de la estabilidad social. } \\
\text { - Defensa del valor de la democracia. }\end{array}$ \\
\hline
\end{tabular}

Resumen de argumentos usados en los debates en el Congreso y en el Senado, en relación de la ley que modifica el Código Civil, para incorporar la figura del matrimonio entre personas del mismo sexo.

En el siguiente cuadro resumimos los argumentos utilizados por el grupo favorable a la reforma, y los grupos opositores. Dicho cuadro categoriza a los argumentos según el nivel de racionalidad de la propuesta de Atienza a la que pertenecen. Sin embargo, no pretendemos ser exhaustivos respecto del uso de cada argumento. Debido a la extensión de los debates, y al número de oradores involucrados, es natural que los argumentos se repitan, por lo que citaremos algunos de sus usos más claros sin perjuicio de que sean encontrados en diversas formulaciones a lo largo de la transcripción de las sesiones en el Congreso y en el Senado.
La tabla presentada resume algunos de los principales argumentos utilizados en las dos instancias de debate, en donde a pesar de la extensión de algunas intervenciones, el debate se centró siempre en algunos puntos altamente polémicos. Lo importante de notar -en contraste con países en los que recién se vienen debatiendo estos temas, como Perú- es que incluso los Congresistas y/o senadores de los grupos más conservadores parecen estar de acuerdo con que un estado de cosas en el que no se permite ningún reconocimiento jurídico a las relaciones de personas del mismo sexo resulta discriminatorio. La divergencia se ubica a un segundo nivel, y consiste en la discusión sobre si la materialización más idónea del mandato de no discriminación es a través del matrimonio o de otras figuras que existen a nivel comparado. El siguiente gráfico resume lo anterior:

Gráfico 1: Estructura de fondo sobre el debate español 2005

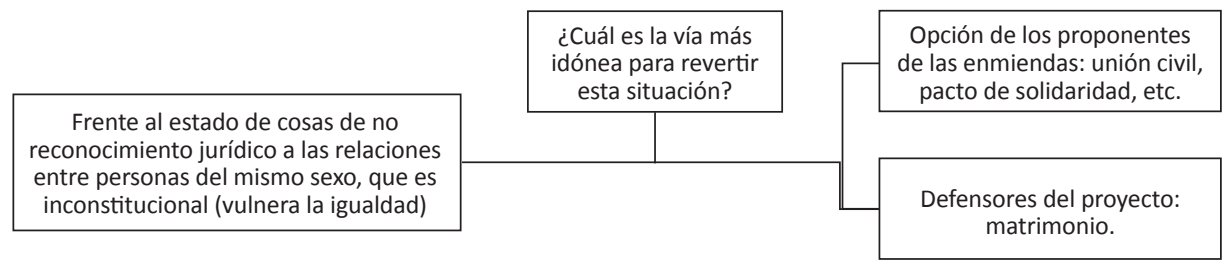

Gráfico 2: Estructura de fondo sobre el debate peruano $2014^{23}$

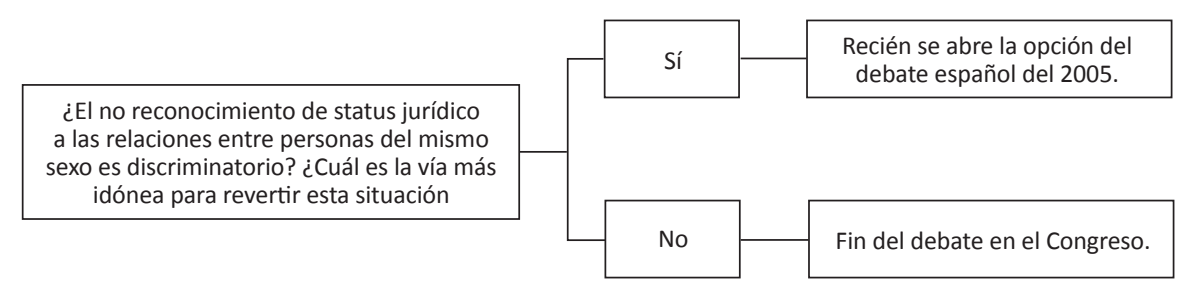

19 Duran i Lleida: "No estoy de acuerdo con que no admitir el matrimonio de personas del mismo sexo es discriminatorio. A mi juicio, nada más lejos de la realidad. Podemos tratar de manera diversa aquello que es diferente; la discriminación vendría por el tratamiento idéntico de realidades distintas". Debate en el Congreso. p. 3774.

20 Duran i Lleida: "[...] porque las discriminaciones que se puedan dar en el tejido social no se evitan mediante la configuración legal de un matrimonio". Debate en el Congreso. p. 3774.

21 Véase, sobre todo, la intervención del Ministro de Justicia, Debates en el Congreso. pp. 3769-3773.

22 Véase, en especial, la última parte de la intervención de la congresista Villarrubia Mediavilla. Debates en el Congreso. p. 3788.

23 Para una excelente revisión del estado de la cuestión en el debate peruano en el 2014, véase MEZA, Carla (relatora). "Informe anual sobre Derechos Humanos de personas trans, lesbianas, gays y bisexuales en el Perú 2013-2014". Lima: PROMSEX. En: http://www.promsex.org/documentacion/publicaciones/item/2382-informe-anual-sobre-derechos-humanos-de-personas-trans-lesbianas-gays-y-bisexuales-en-el-peru-2013-2014.html 
Lo que resulta interesante de notar es que a diferencia del debate peruano, en el que aún no existe pleno acuerdo sobre la pertinencia del debate respecto de la eventual afectación de bienes constitucionales, el acuerdo de fondo español nos permite avanzar hacia un análisis de idoneidad y necesidad en los que nos centraremos en esta parte final.

Wintgens propone que existe un deber de pesar y balancear opciones alternativas en la actividad legislativa. Este deber se extrae de la justificación legisprudencial y demanda el análisis de un conjunto de alternativas. En el presente caso, parecieran existir algunas opciones aparentemente idóneas para lograr la misma finalidad, que es el reconocimiento jurídico de las parejas del mismo sexo. Tenemos al matrimonio, a la unión civil, a la figura del pacto de solidaridad, entre otras. La pregunta a responder es qué elementos diferencian a cada una de estas alternativas, y cuál de todas reporta el mayor beneficio respecto de la finalidad buscada.

En las propuestas de veto del Senado y Pleno, oradores como Casas i Bedós ${ }^{23}$ citan en extenso la opinión del Consejo de Estado -órgano consultivo del gobierno español- para sostener que la ampliación del matrimonio a parejas del mismo sexo supone una alteración institucional que -inferimos- supera en sus perjuicios a los beneficios reportados por la eliminación del trato discriminatorio. Por otra parte, los defensores del proyecto señalan que no existe una vía igualmente satisfactoria, para lograr la finalidad de la total erradicación de la discriminación, que la del matrimonio. La discusión se lleva a cabo entre dos niveles de análisis: el de idoneidad ("qué medida es idónea para la finalidad buscada") y el de necesidad ("qué medidas, siendo idóneas, son necesarias").

Sin embargo, mientras que los tribunales y jueces están premunidos de las herramientas de la ponderación y del tratamiento de colisiones entre principios, el parlamento y senado se encuentra más expuesto a exámenes superficiales de peso y balanceo de alternativas. Analíticamente podemos criticar la poca precisión del debate. Si de lo que se trata es de analizar la medida más idónea para lograr la completa erradicación de la discriminación en el tratamiento de las parejas homosexuales y heterosexuales, la vía matrimonial es la idónea, y las vías alternativas parecen no serlo. Esta respuesta es correcta, salvo que existieran principios que del otro lado, desaconsejen que el matrimonio sea la alternativa elegida. Aquí el argumento se desliza entre apelaciones a la función natural del matrimonio, y razonamientos que ponen en el centro de la discusión al debate sobre la adopción. En esta segunda vía -por cierto más desarrollada a nivel argumental- las instituciones alternativas (unión civil, pacto de solidaridad, etc.) se muestran como idóneas, pues el matrimonio -en esencia-implica la posibilidad de adopción.

\section{CONCLUSIONES}

A continuación, resumimos -muy esquemáticamente-algunas de las principales conclusiones del presente trabajo:

Un trabajo más profundo sobre la racionalidad legislativa demanda un análisis tanto de los debates formales en los plenos del congreso o senado, como de los debates más informales en universidades, medios de comunicación o mítines políticos. Este tipo de estudio permite evidenciar adaptaciones retóricas de los oradores según el tipo de auditorio frente al que se presentan. Asimismo, permite identificar el nivel de flexibilidad de los oradores para escuchar posturas de distintos actores ubicados en la periferia del espacio político-formal.

i. La incorporación de las dimensiones sistémica y de legitimidad permite observar cómo los parlamentos son la bisagra a través de la cual el sistema político se hace cognitivamente abierto a demandas sociales, y evita el recalentamiento del sistema del derecho. Asimismo, nos permite observar cómo las limitaciones o formulaciones legislativas se construyen con una pretensión de legitimidad que consiste en su exhaustiva discusión pública. En el plano ideal ello permite que seamos autogobernados por las propias leyes de las que participamos en su activa discusión y debate.

ii. En concreto, sobre el debate analizado, el modelo de niveles de racionalidad de Atienza permite identificar diversos tipos de argumento. Ello es indispensable para una mejor distinción analítica sobre los reales desacuerdos entre las bancadas, más allá de su ubicación en el espectro político, o de la ideología compartida por sus partidarios.

iii. El acuerdo de fondo sobre la discriminación en la falta de tratamiento jurídico a la situación de las parejas del mismo sexo permitió avanzar sobre las propuestas de solución. Sin embargo, a este nivel, las técnicas parlamentarias de balancing y ponderación son bastante menos desarrolladas que sus equivalentes judiciales. Lo anterior se puede acentuar, además, cuando los parlamentos saben que cuentan con el respaldo y "filtro

24 En especial "Debates del Senado-Pleno". p. 2501. 
de constitucionalidad" de un tribunal que puede revisar la normativa que se apruebe. Ello puede politizar y desconstitucionalizar el debate, lo cual puede perjudicar a grupos vulnerables o que sufren de restricciones para incorporarse al debate público.

\section{BIBLIOGRAFÍA}

\section{Doctrina}

AGUILÓ, Josep. "Cuatro modos de debatir". En: DOXA - Cuadernos de Filosofía del Derecho 36. 2013.

https://doi.org/10.14198/doxa2013.36.10

ATIENZA, Manuel. "Contribución a una teoría de la legislación”. Madrid: Cívitas.1997.

FERNANDES, Celso. "Protestas sociales dentro del derecho". Lima: Fondo Editorial PUCP e Hipocampo. 2012.

GARCÍA AMADO, Antonio. "La filosofía del derecho en Habermas y Luhmann”. Bogotá: Universidad Externado de Colombia. 1997.

HABERMAS, Jürgen. "Teoría de la acción comunicativa", tomo I. Madrid: Taurus. 1999.

OLIVER-LALANA, Daniel. "Normas y razones: un estudio sobre argumentación legislativa". En: GRÁNDEZ, Pedro y Félix MORALES (eds.). "La argumentación jurídica en el Estado Constitucional". Lima: Palestra. 2013.

LUHMANN, Niklas. "Law as a social system". En: Northwestern University Law Review 83.

LUHMANN, Niklas. "Legal argumentation: an analysis of its form". En: The mo- dern law review 58. 1996. https://doi. org/10.1111/j.1468-2230.1995.tb02011.x

LUHMANN, Niklas. "Operational closure and structural coupling: The differentiation of the legal system". En: Cardozo Law Review 13. 1992.

NINO, Carlos. "La Constitución de la democracia deliberativa”. Barcelona: Gedisa. 2009.

SAGER, Lawrence. "Juez y democracia". Madrid: Marcial Pons. 2007.

SIECKMANN, Jan. "Legislation as Implementation of Constitutional Law: A Foundation for the Demand of Legislative Rationality". En: WINTGENS, L.J. y Daniel OLIVER-LALANA (eds.). "The rationality and Justification of Legislation". Suiza: Springer. 2013. https://doi. org/10.1007/978-3-319-00062-6_6

TUSHNET, Mark. "Is congress capable of conscientious, responsible constitutional interpretation?". En: Boston University Law Review 89. 2009.

WINTGENS, L, J. "Legisprudence. Practical Reason in Legislation". Aldershot: Ashgate. 2012. https://doi.org/10.4324/9781315592145

\section{Páginas web:}

MEZA, Carla (relatora). "Informe anual sobre Derechos Humanos de personas trans, lesbianas, gays y bisexuales en el Perú 2013-2014". PROMSEX. En: http://www.promsex.org/documentacion/publicaciones/item/2382-informeanual-sobre-derechos-humanos-de-personastrans-lesbianas-gays-y-bisexuales-en-el-peru-2013-2014.html 